\title{
Blood circulation in the ascidian tunicate Corella inflata (Corellidae)
}

\author{
Michael W Konrad ${ }^{\text {Corresp. } 1}$ \\ 1 none \\ Corresponding Author: Michael W Konrad \\ Email address: michael@scienceisart.com
}

The body of the ascidian tunicate Corella inflata is relatively transparent. Thus the circulatory system can be visualized by injecting high molecular weight fluorescein labeled dextran into the heart or the large vessels at the ends of the heart without surgery to remove the body wall. In addition, after staining with neutral red the movement of blood cells can be easily followed to further characterize the circulatory system. The heart is two gently curved concentric tubes extending across the width of the animal. The inner myocardial tube has a partial constriction approximately in the middle. As in other tunicates, the heart is peristaltic and periodically reverses direction. During the branchial phase blood leaves the anterior end of the heart by two asymmetric vessels that connect to the two sides of the branchial basket. Blood then flows in both transverse directions through a complex system of ducts in the basket into large ventral and dorsal vessels which carry blood back to the visceral organs in the posterior of the animal. During the visceral phase blood leaves the posterior end of the heart in two vessels that repeatedly bifurcate and fan into the stomach and gonads. Blood velocity, determined by following individual cells in video frames, is high and pulsatory near the heart. A double peak in velocity at the maximum may be due to the constriction in the middle of the heart tube. Blood velocity progressively decreases with distance from the heart. In peripheral regions with vessels of small diameter blood cells frequently collide with vessel walls and cell motion is erratic. The estimated volume of blood flow during each directional phase is greater than the total volume of the animal. Circulating blood cells are confined to vessels or ducts in the visible parts of the animal and retention of high molecular weight dextran in the vessels is comparable to that seen in vertebrates. These are characteristics of a closed circulatory system. 
1 Running title: Blood circulation in tunicate $C$. inflata

2

3 Title: Blood circulation in the ascidian tunicate Corella

4 inflata (Corellidae).

5

6

7 Author: Michael W. Konrad

8 Current address: 38 Santa Rosa Ave., Sausalito CA 94965

9 Correspondence to: michael@s scienceisart.com

10

11

12 


\section{Abstract}

14 The body of the ascidian tunicate Corella inflata is relatively transparent. Thus the circulatory

15 system can be visualized by injecting high molecular weight fluorescein labeled dextran into the

16 heart or the large vessels at the ends of the heart without surgery to remove the body wall. In

17 addition, after staining with neutral red the movement of blood cells can be easily followed to

18 further characterize the circulatory system. The heart is two gently curved concentric tubes

19 extending across the width of the animal. The inner myocardial tube has a partial constriction

20 approximately in the middle. As in other tunicates, the heart is peristaltic and periodically

21 reverses direction. During the branchial phase blood leaves the anterior end of the heart by two

22 asymmetric vessels that connect to the two sides of the branchial basket. Blood then flows in

23 both transverse directions through a complex system of ducts in the basket into large ventral and

24 dorsal vessels which carry blood back to the visceral organs in the posterior of the animal.

25 During the visceral phase blood leaves the posterior end of the heart in two vessels that

26 repeatedly bifurcate and fan into the stomach and gonads. Blood velocity, determined by

27 following individual cells in video frames, is high and pulsatory near the heart. A double peak in

28 velocity at the maximum may be due to the constriction in the middle of the heart tube. Blood

29 velocity progressively decreases with distance from the heart. In peripheral regions with vessels

30 of small diameter blood cells frequently collide with vessel walls and cell motion is erratic. The

31 estimated volume of blood flow during each directional phase is greater than the total volume of

32 the animal. Circulating blood cells are confined to vessels or ducts in the visible parts of the

33 animal and retention of high molecular weight dextran in the vessels is comparable to that seen

34 in vertebrates. These are characteristics of a closed circulatory system. 


\section{Introduction}

37 Vertebrates are one of three subphyla in the phylum Chordata. Genomic DNA sequence analysis

38 strongly suggests that the tunicate subphyla, not the apparently more similar cephalochordates

39 are the sister group to vertebrates (Delsuc et al. 2006). Thus the study of tunicates could play an

40 important role in understanding vertebrate evolution from a common ancestor.

41 Ciona intestinalis, a member of the class Ascidiae, has been used in many of the recent studies of

42 embryological development and gene expression, (Stolfi and Christiaen 2012) and it is the

43 subject of many hundreds of publications. In particular, the development of the heart (Christiaen

44 et al. 2009; Davidson 2007; Davidson et al. 2006; Stolfi et al. 2010), and by association the

45 circulatory system, has been the subject of recent reports. The three orders of Ascidiae are

46 differentiated by the morphology of the branchial basket that supports the filter feeding net. Both

47 C. intestinalis and C. inflata are in the order Phlebobranchiata, with a basket vasculature

48 intermediate in complexity in the three orders. However, C. intestinalis is in the family Cionidae,

49 with visceral organs posterior to the branchial basket. Berrill (1955) considers this family

50 primitive because it is "the only family in which viscera continue to occupy the position they

51 held in the beginning (of morphogenesis), ... in all other families the viscera either descends into

52 the stalk or is dislocated along one side of the branchial region". C. inflata, the tunicate described

53 in this study, (Lambert et al. 1981) is in the family Rhodosomatidae, with a digestive track and

54 heart on the right side of the body (van Name-1945).

55 In tunicates motion of the peristaltic heart is often visible, and it is apparent that the direction of

56 pumping reverses periodically, typically every one to three minutes. At present there is no

57 commonly accepted physiological advantage for the reversal, but it may be at the root of the 
58 suspicion that the circulatory system in tunicates is fundamentally different from the vertebrates

59 in which blood always flows in one direction. For example, the heart might cycle in direction to 60 pump blood from one large internal sinus to the other. However, Krieble (1968) estimated the 61 total volume of blood pumped during one directional phase as the product of the volume of the 62 heart and the number of heart beats, and found this volume to be equal to the weight of the 63 animal, far too large to be stored in any internal sinus. This same publication mentioned several 64 citations presenting evidence that in one directional phase the blood completes several complete 65 circulations, and describe the system as closed. There is no experimental evidence for lack of a 66 complete circulation loop from one end of the heart to the other.

67 However, circulation in tunicates is often described as "open" (Davidson 2007; Monniot et al. 68 1991; Passamaneck \& Di Gregorio 2005; Satoh 1994). An "open circulation" usually refers to a specific configuration often seen in crustaceans and insects. Blood is pumped through a vascular network that delivers it to various parts of the body. The blood then percolates around cells of the

71 body tissues to finally collect in a large, pericardial sinus. The heart siphons blood from this

72 sinus to complete the circulation. In an open circulation the term "hemolymph" is preferred over

73 "blood" because the fluid is in direct contact with somatic cells during a large portion of its

74 circulation, and thus is more analogous to lymph than the blood in the closed vertebrate

75 circulation. However, there has been no report of a pericardial blood sinus in any ascidian.

76 The arguably most complete description of the anatomy of an ascidian tunicate is the 123 page

77 monograph Ciona, (Millar 1953), where the circulatory system is described in detail. The ventral

78 end of the heart connects directly to the vessel that runs under the ventral edge of the branchial

79 basket. Blood then flows through many parallel transverse vessels across the branchial basket to 80 empty into the large dorsal vessel and flow back to the visceral, posterior region of the animal. 
81 Since sea water flows across both sides of the branchial basket, blood must always be enclosed,

82 or it would be lost. The ventral, dorsal end of the heart is connected to a bifurcating vessel tree

83 supplying blood to the stomach, ovaries, testes, etc. There is no mention of a pericardial sinus in

84 Millar's work. In some publications circulation in tunicates is said to be open because the blood

85 vessels lack endothelial cells. Citations are not given, but the source is again probably Millar

86 (1953). In this publication reference to endothelial cells and blood vessels consists of one

87 sentence in which the author states that endothelial cells were only found associated with vessels

88 near the heart. There is no mention of criteria used to define endothelial cells, or which vessels in

89 the animal were studied. Thus documentation of the histology of tunicate vessels is rather thin,

90 and certainly deserves more attention. However, an anatomically closed circulatory system

91 which is described as open only because the vessels are permeable or leaky has not been reported

92 in the literature.

93 The present report is the first description of the circulatory system of the ascidian tunicate

94 Corella inflata (Lambert et al. 1981). This animal is more transparent than C. intestinalis, and

95 the circulatory system is clearly visible without the need of surgery to remove the tunic and body

96 wall. Staining with neutral red dye facilitated observation of moving blood cells which defines

97 the circulatory system. In contrast to $C$. intestinalis blood leaves the ventral end of the heart in

98 two vessels which supply the two sides of the branchial basket. Individual cells could not be

99 followed in the peristaltic heart but blood flow and motion of the myocardial heart tube suggests

100 an attachment at a constriction in the middle of the heart.

101 Measurement of blood cell positions in a series of consecutive video frames allowed construction 102 of graphs of cell velocity versus time near the heart. Blood flow is pulsating, falling almost to 
103 zero between a double maximum, which may be a consequence of the constriction in the middle

104 of the heart tube.

105 The body of $C$. inflata is rigid, and does not contract significantly when probed with needles.

106 This insensitivity facilitated injection of high molecular weight dextran into the circulatory

107 system at both ends of the heart and during both directional phases of heart action. Distribution

108 of dye clearly reveals two bifurcating trees of vessels rooted at both ends of the heart. Retention

109 times of the dye in vessels were similar to that seen in vertebrates, and thus the vessels are no

110 more permeable than vertebrate vessels, at least at the blood pressures present in the tunicate.

\section{Methods}

\section{Animal collection and care}

113 Corella inflata HunTSMAN 1912 (Lambert et al. 1981) were collected in a marina in Sausalito,

114 CA, USA (San Francisco Bay) from the side of a floating dock at a depth of $0.1-0.3 \mathrm{~m}$. The

115 present study is the result of observations on 94 animals over a period of 15 months.

116 Tunicates were kept in a seawater aquarium at temperatures of $16-20 \mathrm{C}$ with aeration, and used

117 within 72 hours of collection. During injections and observations animals were submerged in

118 seawater and pinned to a $5 \mathrm{~mm}$ thick gel of silicon (Slygard 184, Global-Industrial Corp) in a $7 \mathrm{x}$

$1197 \mathrm{~cm}$ acrylic box. The top of the animal was typically only 1-3 $\mathrm{mm}$ below the surface of the

120 seawater. No surgery was done to remove the tunic or any other part of the animal.

121 Staining with neutral red

122 Tunicates were stained by immersion in $50 \mathrm{~mL}$ of a $0.1 \%$ solution of neutral red dye (Cynmar

123 Corp.) in seawater for 10 minutes. They were then washed twice in $50 \mathrm{~mL}$ of seawater. 
127 Fluorescent dextran (FITC labeled, 150,000 Daltons, Polysciences, Inc.) at a concentration of 5

$128 \mathrm{mg} / \mathrm{mL}$ in seawater was injected using a 33-gauge $\mathrm{x} 1 / 2$ inch hypodermic needle (TSK

129 STERiJECT PRE-33013) connected by $8 \mathrm{~cm}$ of polyethylene tubing (PE/8, $1.2 \mathrm{~mm}$ ID,

130 Scientific Commodities Inc.) to a $1 \mathrm{~mL}$ plastic tuberculin syringe (McKesson, 102-ST1C) with a

131 total plunger travel of $57 \mathrm{~mm}$. The syringe plunger was directly connected to a screw attached to 132 a microprocessor controlled stepping motor with 200 steps per revolution. Rotation of the screw 133 thus rotated the plunger to overcome static friction that could affect fluid delivery. One

134 revolution advanced the screw $0.85 \mathrm{~mm}$ corresponding to $15 \mathrm{uL}$. Injections were controlled by 135 joystick, with stepper motion recorded to a computer file. Typically 5 to $20 \mathrm{uL}$ were injected 136 over a 3 to 15 second period.

137 Imaging of fluorescent dextran

138 Excitation was accomplished using a single LED (Luxeon V Star Blue, $470 \mathrm{~nm}, 48 \mathrm{~lm}$ ) placed 139 about $40 \mathrm{~mm}$ from the animal. Fluorescence was imaged using a long wavelength pass filter 140 Schott GG495, (UQG Optics Limited) to block reflection of the excitation LED.

141 Image capture and processing

142 A Canon Rebel T3i camera and Canon $100 \mathrm{~mm}$ macro lens mounted on a copy stand were used 143 to obtain images of the entire animal. Higher magnifications, required to follow moving blood 144 cells, was obtained using a Meiji stereoscopic microscope and the Canon camera. Images were 145 processed using Adobe Photoshop software with any image enhancement applied uniformly to 146 the entire frame. The velocity of blood cells was determined by measuring position of a selected 
147 cell in sequential frames from a video. Video files were converted to individual images using

148 QuickTime Player 7 (Apple) and the positions of cells were determined using Photoshop

149 (Adobe). The default configuration of Photoshop gives $\mathrm{x}, \mathrm{y}$ positions to 0.1 pixel, which is useful

150 when one wants to specify the center of an object of 3-16 pixels. There are many other

151 combinations of applications that could obtain similar data. In this report velocity is defined as

152 distance/time, where time is $1 / 30$ second, the time between each video frame. Curves in Figure 6 153 represent a moving average over 5 time points.

\section{Results}

155 Transparency of $C$. inflata facilitates visualization of internal structure if the structure is not itself

156 transparent. Thus optimal observation is achieved by using a dye to create contrast. Neutral red is

157 most commonly used in tissue culture as a stain specific for living cells, where it is transported

158 into lysosomes to give the culture a red-pink color (Borenfreund \& Puerner 1984), while also

159 staining nuclear chromatin (Espelosin \& Stockert 1982). Because it is non-toxic and bound

160 complexes are stable it has been used to stain entire marine invertebrates for up to 7 days (Drolet

$161 \&$ Barbeau 2006). In the present study neutral red was used as a relatively non-specific stain for

162 blood cells which were identified by motion. Moving blood cells define the vessels, channels or

163 ducts that distribute blood, and the velocity of the cells reveals the dynamics of the circulatory

164 system. Alternatively, injection of fluorescent dextran into the circulatory system while the heart

165 is pumping defines the distribution of blood throughout the vascular system.

166 In this report the terms "vessel" and "vascular" are used only to specify pathways, channels or

167 ducts through which blood flows. There is no intent to suggest that a "vessel" has the histology

168 of a blood vessel in a vertebrate, as the methods and equipment used in this study do not resolve

169 structure at this scale. 


\section{Structure of the tunicate and location of major blood vessels}

170 The right and left sides of a typical C. inflata stained with neutral red are seen in Fig. 1A and B

171 respectively, with labeled diagrams below each photograph. Tunicates have an inner cylindrical

172 branchial basket attached along the ventral edge, the endostyle, to the outer cylindrical body.

173 Water is pumped into the oral siphon and out of the atrial siphon by cilia in the branchial basket.

174 Food is trapped in a mucus net produced by the endostyle, moved by ciliary action across the

175 interior of the branchial basket and then drawn down into the stomach. C. inflata has an enlarged

176 exit siphon, which in this image contains fecal matter, but is also used to contain and protect the

177 tunicate "tadpoles". The endostyle is the dark band along the ventral side of the animal, the

178 orange band in the diagram. The stomach, ovaries, and testis make up the dark mass at the

179 bottom of the tunicates. The heart is the vertical band along the upper edge of the visceral mass.

180 In C. inflata two large blood vessels exit the anterior (ventral) end of the heart and pass to the

181 middle of each side of the branchial basket where they repeatedly branch and merge into a

182 rectangular net of vessels. One vessel connects directly to the middle of the right half of the

183 basket, Fig. 1A, while the other vessel passes over the endostyle and connects to the left side of

184 the branchial basket, Fig. 1B. The approximate gross volume (all interior space including sea

185 water) of the tunicate is estimated to be $2700 \mathrm{~mm}^{3}$ from the photograph and assuming the animal

186 is a solid trapezoid with constant thickness.

\section{Global pattern of blood flow}

188 Moving blood cells define the functional vessels (Fig. 2 and Video 1). The peristaltic heart of $C$.

189 inflata, like other tunicates, reverses the direction of pumping every few minutes. During the

190 branchial phase of heart action blood is drawn from the viscera into the posterior end of the heart 
191 and then pumped from the anterior end into the branchial basket via two vessels. One vessel

192 leaves the heart and is seen to join the branchial basket on the right side of the animal but the

193 vessel that carries blood to the left side of the branchial basket cannot be seen in the perspective

194 of Fig 2. Blood that enters the middle of the right side of the branchial basket flows in a

195 rectilinear network to vessels at both the dorsal and ventral edges of the basket, and then flows in

196 a posterior direction toward the viscera to complete the circulation. A small fraction of blood

197 leaving the heart supplies the mantle and tunic, but this vessel system is not visible in the focal

198 plane of Fig. 2 and Video 1.

199 Video 1 demonstrates the ability of blood cell movement to identify the circulation system but it

200 only provides information about a limited region of the animal. In order to build the more

201 complete model of circulation described in the text it was necessary to focus at different planes,

202 examine various fields of view, rotate the animal to see regions that are obscured by stained

203 structures, and examine many animals. A dramatic feature of Video 1 is the near synchronous

204 movement of pulsating blood cells throughout the animal as the heart beats. This implies both

205 that the output of the heart is pulsatory and that the phase is approximately constant throughout

206 the animal.

207 Injection of fluorescent dye into the circulatory system

208 Injection of the non-binding tracer, fluorescent dextran, into the circulation allows direct

209 visualization of the heart and the larger vessels. However, the distribution of viscera is laterally

210 asymmetric, and hides much of the circulation in the left side of the tunicate. Thus only the right

211 side is shown in this study. Dextran was injected into the posterior end of the heart pumping in

212 the branchial phase. The dye then flowed into a vessel leaving the anterior end of the heart and 
213 into the right side of the branchial basket where it filled a rectilinear vascular network (Fig. 3A).

214 Dextran flowing to the left half of the branchial basket cannot be seen in this view.

215 In a second experiment dextran was injected into a vessel at the anterior end of the heart

216 pumping in the visceral phase. The dye was injected at the juncture of the two large vessels that

217 leave the heart from the posterior end (Fig. 3B). One vessel bends sharply in the ventral direction

218 and then branches to supply the stomach and gonads. The other vessel branches toward the

219 intestine and the main dorsal vessel. The injection also inadvertently introduced fluorescent

220 dextran into the pericardial sac that encompasses the inner heart tube. Thus the heart appears to

221 be filled with fluorescent dextran even though at the time this image was obtained most of the

222 fluorescent dextran in the interior heart tube has moved to the visceral region.

223 As with other ascidians the heart consists of two concentric cylinders fused at the ends (Millar

224 1953). The surface of the outer heart tube is relatively static, and thus does not directly

225 participate in the pumping process. The pericardial space between the tubes is filled with fluid.

226 Fluorescent dextran injected into the pericardial space of the $C$. inflata heart clearly defines the

227 tube enclosing the heart (Fig. 4). Dextran remained in the pericardial space during a 24-hour

228 period when the heart was beating at its normal rate. Thus, this space is quite isolated from the

229 rest of the animal.

230 Heart structure and function

231 The $C$. inflata heart reverses the direction of peristaltic contractions periodically as has been

232 observed in all other tunicates. Constrictions traverse the heart in an average of 1.4 seconds and

233 approximately every 4 minutes, or 180 beats, the direction of movement reverses (Table 1). 
234 The inner cylindrical tube contracts and twists progressively along its length to produce a

235 moving constriction that forces the blood from one end to the other. The flow of stained blood

236 cells enables movement of the inner heart tube to be followed in some detail. As one peristaltic

237 constriction approaches the end of the heart, another constriction forms at the other end, and thus

238 the heart tube is never open. The constriction that moves the blood through the heart tube does

239 not appear cylindrically symmetric but is rather a twist in folds of the myocardium. About

240 halfway along the heart tube is a stationary pinch of about half the average diameter. As the twist

241 approaches this pinch, blood is forced through and a new fold appears on the other side with

242 mirror image twist. Thus the pinch does not appear to function as a valve. The anterior half of the

243 heart, the heart-tube folds, and the vessel from the heart to the right side of the branchial basket

244 is seen in Fig. 5A, and diagrammed in Fig. 5B. Video 2, which has the same field of view and

245 orientation as Fig. 5, documents the movement of the heart myocardial folds.

246 While rapidly moving stained blood cells can be seen in the heart, the high velocity generates

247 streaks in video frames, and cells come in and out of focus due to the complex flow pattern.

248 However, it was possible to follow the position of individual blood cells in a large vessel about 1

$249 \mathrm{~mm}$ anterior to the heart as seen in Fig. 5, and thus to determine the velocity versus time profile

250 (Fig. 6). Two cells were followed through two heart beats while they moved about $2 \mathrm{~mm}$. The

251 velocity versus time curves are approximately sinusoidal with a period slightly more than a

252 second, consistent with Table 1. There is a reproducible small dip in the region of maximum

253 velocity. The velocity is always positive, i.e. cells always move in the direction of the heartbeat,

254 but the minimum velocity is only about a tenth the maximum value.

255 In a typical animal (Fig. 1), the heart is about $12 \mathrm{~mm}$ long, and a constriction moves through the

256 heart every 1.4 seconds, giving an average velocity of $9 \mathrm{~mm} \mathrm{~s}^{-1}$. The peak velocity of about 2 
$257 \mathrm{~mm} \mathrm{~s}^{-1}$ in the vessel just anterior to the heart (Fig. 6) is thus several-fold lower than the velocity

258 of the heart constriction. However, this is but one of two large vessels the heart empties into, and

259 the total cross sectional area of these vessels can only be crudely estimated. Blood cell velocities

260 in three small vessels in the mantle at the extreme anterior end of the animal are much smaller

261 than seen in the large vessel (Fig 7). These velocities appear stochastic, with an average of about

$2620.3 \mathrm{~mm} \mathrm{~s}^{-1}$, but all are positive (move in the same direction) throughout several heartbeats.

\section{Blood flow in the branchial basket}

264 The cylindrical branchial basket occupies a major portion of the tunicate. As seen in Fig. 3A,

265 blood flows from the ventral end of the heart to the middle of each side of the branchial basket.

266 On the interior of the basket a rectangular network provides support and distributes blood (Fig.

267 8A). Blood then flows into an exterior layer perforated with pairs of spiral stigmata resembling

268 the Ionic volutes of Greek columns (Fig. 8B). Cilia line the inside edges of the stigmata, but can

269 only be seen by their motion. Fluorescent dextran injected into the blood reveals the structure of

270 the duct containing the stigmata in low resolution (Fig. 8C). Blood cells could be seen moving in

271 the area immediately adjacent to and between the stigmata. This is consistent with stigmata being

272 openings through a double layer of cells, and blood circulating in the space between these cell

273 layers.

274 Blood from the branchial basket is collected by vessels along the ventral and dorsal edges of the

275 branchial basket to flow to the posterior of the animal (summarized in the schematic of Fig. 2).

276 When the heart reverses direction the direction of blood flow in all parts of the animal reverses.

\section{Discussion}


278 Distribution of injected dextran and the flow of blood cells are complementary methods, dextran

279 defining the larger vessels and their permeability while cell flow also reveals small vessels and

280 blood velocity. Circulation of blood can be followed through most of the tunicate body.

281 Blood leaves the anterior end of the heart via two vessels supplying the two sides of the

282 branchial basket. The blood is then flows through transverse tubes into ducts containing the

283 stigmata. Blood is collected by one large vessel on the ventral and another on dorsal side of the

284 body to flow back into the visceral region. Blood is collected from the visceral region by a

285 branched vascular tree merging into two large vessels entering the posterior end of the heart. It

286 has not been possible to trace blood flow through the entire visceral region, specifically in the

287 space between the posterior ends of the dorsal and ventral vessels and the ends of the vascular

288 tree that feeds blood into the heart. The major difficulty is the density and opacity of the organs

289 in this region. It is always possible that blood does not flow through clearly defined vessels in the

290 viscera, but rather through a network of cavities as seen in the liver and spleen of vertebrates

291 (Richardson \& Granger 1984).

292 Ruppert (1990) has described transport of sea water by the neural gland across the wall of the

293 pharynx into the vasculature of the ascidian Ascidia interrupta, and suggested that the neural

294 gland regulates vascular volume after contractions, "squirts". However, Corella inflata does not

295 contract often, and in the present investigations there were no contractions.

296 Vessel structure

297 The two large vessels that leave the anterior end of the heart follow asymmetric paths to the two

298 sides of the branchial basket and do not appear to be associated with any of the symmetric

299 structures in that region. In that sense they are independent structures. 
300 The molecular integrity of vessels can be inferred by the rate infused fluorescent dextran leaks

301 into surrounding tissue. A halo of fluorescence can be seen around the large vessels supplying

302 blood to the branchial basket about 5 minutes after the start of the injection (Fig. 3A). However,

303 since these vessels supply blood to smaller vessels much of the halo seen at this resolution could

304 represent distribution, not leakage. Dextran flowing into the posterior region (Fig. 3B) remains in

305 the major vessels for more than 6 minutes after the injection. Dextran of this molecular size has

306 been used to follow plasma leakage in comparable times from vessels in the hamster cheek

307 pouch induced by leukotrienes (Dahlen et al. 1981), and leakage could be compared in normal

308 and treated animals. In the present report we can just show that tunicate vessels contain dextran

309 for at least five minutes, a time comparable to vertebrate vessels. However, blood pressure in

310 tunicates is approximately 100 times lower than that in vertebrates (Jones 1985), thus absolute

311 tunicate vessel permeability could well be much larger than found in vertebrates and still give

312 comparable leakage rates.

\section{Heart structure and function}

314 The slightly curved cylindrical heart pericardium extends across most of the right side of the

315 body at the anterior edge of the visceral region. While clearly visible on the right it is mostly

316 obscured by the visceral mass when viewed from the left. Concentric with the pericardium is the

317 inner myocardial heart tube, which contains the blood and joins with two large vessels at each

318 end. If homologous with the heart of $C$. intestinalis, the myocardial tube is connected to the

319 pericardial tube by a raphe which extends along the anterior length of both. A peristaltic heart

320 branching into two vessels at each end, is the canonical structure of the vertebrate embryonic

321 heart soon after the heart tube is formed (Gilbert 2010; Santhanakrishnan \& Miller 2011). 
322 The heart myocardium moves the blood using a constriction, or twist, that travels along the tube

323 with a velocity of about $9 \mathrm{~mm} \mathrm{~s}^{-1}$. As one twist approaches one end of the tube a new twist forms

324 at the other end, and thus there is always at least one twist to constrain blood flow. The shape of

325 the myocardial heart tube between twists is an elongated ellipsoid which is distorted as it passes

326 through the median constriction. Thus when blood flows into vessels of fixed diameter at the

327 ends of the heart its velocity is not constant, but rather has a quasi-sinusoidal shape similar to the 328 profile of the blood bolus in the heart.

329 The performance of the tunicate peristaltic heart is quantitatively different from the "technical" 330 peristaltic pumps used in industry, laboratories and hospitals (Manner et al. 2010). The flexible 331 tubes of these pumps have a constant diameter which is compressed by a succession of rollers

332 that are typically separated by several times the diameter of the tubing. Movement of the rollers 333 then produces a constant flow rate that is only transiently modified when the roller leaves the 334 tubing.

335 The pulsating movement of blood cells in the branchial phase of blood motion appears to be in phase 336 throughout the animal, Video 1 . This means that the pulse wave velocity (PWV) is large compared to 337 the size of the animal and the heart beat rate (Zamir 2000). The PWV has been studied in humans 338 because of its potential for diagnosis of abnormal artery rigidity. PWV values in humans are in the range 339 of $2-4 \mathrm{~m} / \mathrm{s}$ (Yamashina et al. 2002), so that the pulse in even distant parts of the body are out of phase 340 by only a fraction of a heart beat cycle. Since the tunicate is about 100 times smaller than a human, the 341 diameters of the vessels are correspondingly smaller, and the heart rate is only 2-3 times as rapid, one 342 would expect blood cell movement to be essentially synchronous throughout the system unless blood 343 vessels were two orders of magnitude more flexible. It is thus not unexpected that blood cells pulse in 344 synchrony throughout the tunicate, consistent with a closed, fairly rigid circulatory system. 
345 In one of the two large vessel near the anterior end of the heart blood cells were observed to have

346 a semi-sinusoidal velocity-time curve with a maximum of $2.2 \mathrm{~mm} \mathrm{~s}^{-1}$. The fish is perhaps the

347 best vertebrate comparison, and a blood velocity of $1.7 \mathrm{~mm} \mathrm{~s}^{-1}$ has been measured in the aorta of

348 a 5 day-post fertilization Zebrafish (Watkins et al. 2012). Zebrafish of this age are only about 2.5

$349 \mathrm{~mm}$ long (Parichy et al. 2009) compared to a typical length of $30 \mathrm{~mm}$ for the Corella in this

350 study, and blood velocity increases as the fish grows. However, blood velocities in the tunicate

351 and fish are at least roughly comparable.

352 The peak velocities of blood in vessels proximal to the heart will not necessarily be equal to the

353 velocity of the heart twist, because the total cross-sectional area of these vessels may not be

354 equal to the maximal cross sectional area of the heart. However, it is difficult to measure the

355 diameters of the heart and vessels with much accuracy since the edges do not appear sharp in the

356 microscope. In addition, since the area of a tube is proportional to the square of its diameter,

357 errors in area are twice those of the diameter measurements.

358 As vessels extend from the heart and repeatedly bifurcate the total cross section must increase,

359 since a decrease in blood cell velocities is seen in videos. Since retention of fluorescent polymer

360 indicates that the vasculature is not leaky, at least for periods of a few minutes, the decrease in

361 cell velocities is just a consequence of conservation of blood volume. In a variety of biological

362 circulatory systems an increase in total cross section after bifurcation of a vessel follows

363 Murray's law, which states that the sum of the cubes of diameters of the branches equals the

364 cube of the diameter of the trunk vessel. In the very compact circulatory tree of the tunicate it

365 seems likely that the velocity of blood decreases almost continuously from the time it leaves the

366 heart. The velocities of individual blood cells graphed in Figure 6a are not the velocities of blood

367 flowing past a fixed position in a vessel, but rather a Lagrangian plot of the velocity of a cell as it 
368 travels along a flow path in a vasculature with a gradient of decreasing blood velocity. Thus the

369 velocity of these cells is a function of both the phase of the heart beat and the position of the cell

370 along the vessel. Each cell described by Figure 6 is followed through two heart beats, and the

371 peak velocity in the second beat always lower than that in the first, presumably because the cell

372 is then farther away from the heart and the cross sectional area is larger. If the velocity of the

373 cells is always decreasing, even the shapes of the velocity versus time curves graphed in Fig. 6

374 have been distorted compared to a curve measured at a fixed position. Thus averages of these

375 peak velocities have little meaning since the cells are at different positions in the vessel. While

376 videos of cell movement provide an exciting possibility to map blood flow throughout the

377 animal, computer implemented image acquisition and analysis is clearly required for any

378 comprehensive quantitative study.

379 In vessels distant from the heart with a small diameter, for example in the body wall, Figure $6 \mathrm{~b}$,

380 maximum velocities range from 0.7 to $0.5 \mathrm{~mm} \mathrm{~s}^{-1}$. Cell motion is often very irregular and

381 velocities do not follow a smooth curve in synchrony with the heartbeat. This irregularity is not

382 surprising since the cells are seen to frequently hit and sometimes momentarily stick to the vessel

383 wall, also impeding movement of neighboring cells. This behavior is not unlike the flow of red

384 blood cells through the capillaries of vertebrates and the velocity of blood cells is an unreliable

385 measure of fluid velocity.

386 The myocardial tube has a fixed constriction approximately in the middle. A change in the twist

387 of the heart tube as the peristaltic contraction passes through this constriction indicates it is a real

388 anatomical feature. It does not function as a valve as blood cells pass through it freely throughout

389 the heart cycle. Hecht (1918) reported seeing a constriction, or "node", in the middle of the heart

390 of Ascidia atra, a tunicate in the same order as $C$. inflata. He described the node in detail, and 
391 stated it was a "real landmark in the structure". However, Goodbody, observing the heart of

392 Corella willmeriana, which is likely to be the same species as $C$. inflata, also observed an

393 apparent constriction in the heart but believed it was not a real anatomical structure but just a

394 "shifting of the position the raphe", (Goodbody 1974). He referred to Hecht's report and stated

395 that Hecht also thought the node was just a shifting of the raphe. In my opinion this is not an

396 accurate description of Hecht's claims.

397 Microscope optics, illumination, and ascidian species used by Hecht, Goodbody, and the present

398 author are all different, and the two previous authors did not stain the animals. However, I have

399 seen the "node" in all my observations, and along with Hecht believe it is a "real landmark".

400 Since the velocity profile of blood cells in the vasculature is a reflection of the shape of the blood

401 bolus in the heart I propose that the dip in velocity seen at the maximum in all the velocity

402 profiles of Fig. 6 is a consequence of this constriction.

403 The constriction divides the heart in two sections, which might suggest either an evolution

404 toward or regression from a two chambered heart homologous to the two chambered heart of the

405 fish. Davidson et al. (2006) demonstrated a requirement for Ets1/2, a transcriptional effector of

406 receptor tyrosine kinase signaling, in formation of the heart tube in C. intestinalis. They also

407 constructed a transgenic $C$. intestinalis producing a constitutively activated form of the Ets $1 / 2$

408 gene product in heart and tail precursor cells. They found an "unexpected phenotype" was

409 produced, an animal with a functional two compartment heart. Presumably it was unexpected

410 because a mere increase in a factor generated a different structure, not just one of a different size

411 or proportion. This suggests an unused, or underused genetic scaffold for a two chambered heart

412 is endogenous to C. intestinalis, and the increase in Ets $1 / 2$ acted on this scaffold to produce the 
413 new heart structure. The same scaffold could well be present in $C$. inflata but in that environment

414 produces a single chambered heart with a middle constriction.

415 Blood flow in the branchial basket

416 The peripheral wall of the branchial basket consists of two parallel layers of cells, penetrated by

417 openings, the stigmata, through which water is pumped by a stack of seven layers of cilia bearing

418 cells along the circumference of the stigmata (Burighel \& Cloney 1997). The wall of the

419 branchial basket is thus homologous to the double-layered lamella (Olson 2002), that constitutes

420 the gills of fish. However, in the fish the layers are closer together, are held in place by pillar

421 cells, and of course are not penetrated by stigmata.

422 Blood, supplied by transverse ducts, seen in Fig 8A, flows between the walls of the basket, Fig

$4238 \mathrm{C}$, and is collected by vessels along the ventral and dorsal edges of the basket. Thus, the shapes

424 of the stigmata, which are spiral in C. inflata, define not only the flow of water through the

425 basket, but also the flow of blood within the walls of the branchial basket. In C. inflata the two

426 vessels from the heart to the middle of the sides of the basket, the transverse ducts, and finally

427 the hollow walls of the branchial basket and the stigmata should all be considered part of the

428 pattern of blood circulation characteristic of the species.

429 Comparison with the circulatory system of Ciona intestinalis

430 In contrast to C. inflata, no constriction in the middle of the heart tube has been described in C.

431 intestinalis, however it would be difficult to observe since the entire heart bends sharply in the

432 middle more than 90 degrees. The stigmata of $C$. intestinalis are thin rectangles aligned with the

433 longitudinal bars, as contrasted to the double curved stigmata of $C$. inflata. Millar did not 
434 describe the double walls of the branchial basket in C. intestinalis, but may have just been

435 unaware of this feature.

436 In Text-Fig. 6, pg 57 Millar (1953) shows blood flowing from the heart through the ventral

437 endostyle, across the branchial basket, and back down the dorsal vessel to the visceral region, a

438 sequence that suggests a circulation. He cites Skramlik (1929) as finding that there is "true

439 circulation of the blood", and does not state opposition to this conclusion.

440 In C. intestinalis blood flows from the anterior end of the heart directly into the vessel running

441 along the ventral edge of the endostyle. In C. inflata it is possible that early in the metamorphosis

442 from the tadpole the heart was also at the midline but had vessels established to the middle of

443 each half of the branchial basket. The heart then migrated from midline to the right half of the

444 animal, passing over the endostyle to its final position and dragging the left branchial vessel

445 along with it. Berrill (1936) describes the extensive migration of the heart relative to the viscera

446 during metamorphosis of several genera of Ascidians; unfortunately Corella was not mentioned.

\section{Open or closed, tidal or circulating}

448 Discussion of blood circulation in tunicates is complicated by potential confusion between two

449 distinct characteristics of a circulatory system and by the use of terms that are not neutral with

450 respect to these characteristics.

451 In an open circulatory system blood is constrained to vessels for a relatively short distance after

452 leaving the heart, while in the remaining part of the circulation it freely percolates through the

453 cells. Since tissues are directly bathed in blood, it is typically called hemolymph. In a closed

454 system blood is confined to a distinct vascular space. Thus the use of "blood" in the title of this

455 report suggests the author believes circulation in $C$. inflata is closed and confined to vessels. 
456 However, while blood in a closed circulation is confined, water, blood proteins and white blood

457 cells percolate at various rates through vessel walls to become lymph. Thus, open versus closed

458 may best be considered a quantitative parameter describing the degree or rate blood is filtered

459 before reaching the cells. In this report the persistence of dextran in the vasculature of C. inflata

460 is comparable to that seen in the closed circulation of vertebrates and thus circulation in this

461 tunicate can also be described as closed.

462 The endothelial cells that line or constitute the blood vessels of vertebrates are considered the

463 essential barrier between blood and lymph (Reiber \& McGaw 2009). Since most invertebrates do

464 not have endothelial cells, and Millar reports that endothelial cells are found only near the heart

465 in the tunicate $C$. intestinalis, one might conclude that circulation in tunicates must be open.

466 However, Reiber and McGaw argue that the open-closed character of circulation is a continuum, 467 note that some invertebrates that lack endothelial cells have a functional closed circulation, and

468 that the invertebrate cephalopod molluscs have a vascular system lined with endothelial cells 469 (Reiber \& McGaw 2009).

470 Circulation can be used as a generic term for any fluid flow. However, used more specifically in 471 physiology blood circulation is a flow that moves around a circuit. Blood circulates through a 472 network of circuits in vertebrates. The hearts of tunicates, including C. inflata, pump in one 473 direction for a period and then reverse for a similar time. An early text on tunicates states: "blood 474 does not go around a true circuit but shuttles back and forth through the heart" (Newman 1939).

475 This quote describes a tidal or reciprocating flow, where blood is pumped from one compartment 476 to another and then back through the same path, not around a closed circuit.

477 However, observation of blood cells in C. inflata reveal they move in circuits, as seen in 478 Video 1, although the flow in all the circuits change direction when the heart changes its 
479 pumping direction. The apparent circulation-tidal contradiction is resolved in the case of $C$.

480 inflata by consideration of the volume of blood pumped by the heart during one directional

481 phase. The inter heart tube has a diameter of about $1.6 \mathrm{~mm}$ and length of $12 \mathrm{~mm}$, to give a

482 volume of $24 \mathrm{~mm}^{3}$. While the heart reversal pattern is likely to be quantitatively dependent on

483 age and size of the animal, water temperature and oxygen concentration, and other variables, the

484 data presented in Table 1 is sufficient to rule out tidal flow. The average number of beats for one

485 cycle is about 270 , and thus the average volume pumped in one cycle would be $6480 \mathrm{~mm}^{3}$ if the

486 heart functioned as a piston moving down a tube with constant diameter. However, as described

487 previously the space carrying the blood is more like an elongated oval between the two twists in

488 the heart tube. Thus the actual volume pumped in one cycle is likely to be several-fold lower.

489 The total volume over the 4 minutes the heart pumps in one direction is larger than the total

490 volume of the animal (tissue and enclosed water), and thus much larger than the total volume of

491 blood. Thus, blood must circulate in this tunicate in the strict sense, it just circulates in opposite

492 directions during the two directional phases of heart action. A similar calculation and conclusion

493 has been made by Kriebel (1968) for circulation in the tunicate Ciona intestinalis.

494 If reversal of blood flow is not required by the anatomy of the circulatory system, there may be a 495 physiological advantage in periodic reversal. One possibility is that the gradient of oxygen and

496 nutrients along the circulation pathway is sufficiently steep that tissues in the second half of the

497 circulation loop do not receive optimal levels. Periodic reversal would provide a more uniform

498 delivery, and Ruppert et al. (2004), on page 946 suggest this possibility. In addition, the

499 peristaltic heart could alter the ratio of beats in one versus the other direction, and thus

500 compensate for changes in nutrient or oxygen supplies and demands. Despite some promising 
501 studies that suggest environmental modification of the reversal patterns, e.g. (Ponec 1982), there

502 is still no generally accepted model for the function of heart reversals.

\section{Comparison to arthropods}

The circulatory system of the invertebrate $C$. inflata tunicate can be compared to the invertebrate arthropods; aquatic crustaceans and terrestrial insects. In arthropods the heart typically consists of segments of the large dorsal vessel that sequentially contract (in peristalsis) to pump blood (hemolymph) from a sinus into vessels extending into each segment of the body. The blood then percolates through the body to return to the sinus (Brusca \& Brusca 2002). In contrast, in $C$. inflata the heart is a single tube which extends diagonally across the entire body. While there is a partial constriction in the middle of the heart, there are no vessels at that location that provide an exit or entrance for blood. When pumping in the branchial direction it obtains blood from a vascular tree that extends into the viscera, and delivers blood to both sides of the branchial basket via another vascular tree, then returning to the visceral region by dorsal and ventral vessels. There is no evidence of a sinus that supplies blood to the heart.

However, a similarity between $C$. inflata (all tunicates) and arthropods is a much lower density of cells in blood compared to vertebrates. The packed cell density in vertebrates is typically close to 30 percent, with most cells being erythrocytes that carry oxygen from air filled lungs to muscles preforming mechanical work. However, in both tunicates and arthropods the cell density in blood is about ten-fold lower. No oxygen carrying molecule analogous to hemoglobin has been identified in the tunicates and thus the oxygen carried by blood is approximately the amount carried by water (Goodbody 1974). However, the blood of many species of arthropods contains the oxygen binding protein hemocyanin. 
In insects, air filled vessels, the tracheal system, supplies oxygen from the atmosphere directly to muscles. In the larger crustaceans hemolymph flows through body gills, often associated with appendages that move to increase both blood and water flow. In tunicates, the major cells performing mechanical work are the cilia surrounding the stigmata, which pump water through the branchial basket and the mucus feeding net. However, these cells are in direct contact with sea water, the source of oxygen for marine organisms.

Only the low blood cell density in $C$. inflata made it possible to determine position versus time for individual blood cells, as seen in Fig. 6. A high cell concentration, comparable to that seen in vertebrates, would present a confusing scene of overlapping cells frequently hiding each other as they changed depth in the ensemble.

\section{Evolutionary relationships}

504 DNA sequences of 18S rDNA genes suggest that the genera Ciona and Corella are as closely 505 related as the two extremely similar Ciona species intestinalis and savignyi (Stach \& Turbeville 506 2002). However, the complete genome sequences for C. intestinalis and C. savignyi suggested 507 that the these two tunicates have diverged from a common ancestor at a time approximately 508 equal to the divergence of the chicken and human (Berna et al. 2009). Thus current analysis of 509 DNA sequences is not particularly useful in understanding the differences in topology of the 510 blood circulation between Ciona and Corella. We will need the complete sequence on C. inflata 511 and better guidance on what specific parts of the genomes to compare to understand the genetic 512 basis of the circulatory system.

513 A comprehensive histological study of the blood vessels in ascidians, e.g. C. inflata and $C$.

514 intestinalis, would certainly extend our understanding of the circulatory systems of tunicates and 515 thus the evolutionary relation to vertebrates. An obvious question is whether there is any 
516 histological or molecular difference between vessels anterior and posterior to the tunicate heart.

517 If so this would suggest that the common ancestor to both vertebrates and tunicates had a

518 unidirectional blood flow. The fact that blood circulates in a closed system in tunicates as it does

519 in vertebrates suggests but does not prove evolutionary similarity. The fact that the two

520 circulatory systems are analogous does not necessarily mean they are homologous (Mayr 2001).

\section{References}

Berna L, Alvarez-Valin F, and D'Onofrio G. 2009. How fast is the sessile ciona? Comp Funct Genomics 2009:e875901:875-901. 10.1155/2009/875901

Berrill NJ. 1936. II-Studies in tunicate development, Part V-the evolution and classification of ascidians. Phil Trans $R$ Soc Lond B 226:43-70.

Berrill NJ. 1955. The Origin of Vertebrates. London: Oxford University Press.

Borenfreund E, and Puerner JA. 1984. A simple quantitative proceedure using monolayer cultures for cytotoxicity assays (HTD/NR-90). J Tissue Cult Meth 9:7-9.

Brusca RC, and Brusca GJ. 2002. Invertebrates. Sunderland, MA: Sinauer Associates.

Burighel P, and Cloney RA. 1997. Urochordata: Ascidiacea. In: Harrison FW, and Ruppert EE, eds. Hemichordata, Chaetognatha, and the invertebrate chordates. New York: WileyLiss, 221-347.

Christiaen L, Wagner E, Shi W, and Levine M. 2009. The Sea Squirt Ciona intestinalis. Cold Spring Harbor Protocols.

Dahlen S-D, Bjork J, Hedqvist P, Arfors K-E, Hammarstrom S, Lindgren J-A, and Samuelsson B. 1981. Leukotriens promote plasma leakage and leukocyte adhesion in postcapilary venules: In vivo effects with relevance to the acute inflammatory response. Proc Natl Acad Sci (US) 78:3887-3891.

Davidson B. 2007. Ciona intestinalis as a model for cardiac development. Semin Cell Dev Biol 18:16-26.

Davidson B, Shi W, Beh J, Christiaen L, and Levine M. 2006. FGF signaling delineates the cardiac progenitor field in the simple chordate, Ciona intestinalis. Genes and Development 20:2728-2738.

Delsuc F, Brinkmann H, Chourrout D, and Philippe H. 2006. Tunicates and not cephalochordates are the closest living relatives of vertebrates. Nature 439:965-968.

Drolet D, and Barbeau MA. 2006. Immersion in neutral red solution as a mass-marking technique to study the movement of the amphipod Corophium volutator. J Crust Biol 26:540-542.

Espelosin RH, and Stockert JC. 1982. Neutral red fluorescence of chromatin: specificity and binding mechanism. Z Naturforsch C 37c:139-141.

Gilbert S. 2010. Developmental biology. 9th ed. Developmental biology 9th ed. Sunderlan MA: Sinauer Associates, Inc., earliest stage of heart development- pg 453: Fig 412.458 Cardiac looping and chamber formation; panelA, day 421.

Goodbody I. 1974. The Physiology of Ascidians. In: Russell SFS, and Younge SM, eds. Adv Mar Biol. London: Academic Press, 1-149. 
Hecht S. 1918. The physiology of Ascidia Atra Leuseur, III. The blood system. Am J Physiol 45:157-187.

Jones HD. 1985. Vascular and pericardiac pressures in Ascidiella aspersa (Urochordata: Ascidiacea). J Mar Biol Ass UK 65:911-922.

Kriebel ME. 1968. Studies on cardiovascular physiology of tunicates. Biol Bull 134:434-455.

Lambert G, Lambert CC, and Abbott DP. 1981. Corella species in the American Pacific Northwest: distinction of C. inflata Huntsman, 1912 from C. willmeriana Herdman, 1898 (Ascidiacea, Phlebobranchia). Canadian Journal of Zoology 59:1493-1504.

Manner J, Wessel A, and Yelbuz TM. 2010. How does the tubular embryonic heart work? Looking for the physical mechanism generating unidirectional blood flow in the valveless embryonic heart tube. Dev Dyn 239:1035-1046.

Mayr E. 2001. What Evolution Is. New York: Basic Books.

Millar RH. 1953. Ciona: The University Press of Liverpool.

Monniot C, Monniot F, and Laboute P. 1991. Coral Reef Ascidians of New Caledonia. Paris: Orstom.

Newman HH. 1939. The Phylum Chordata. Biology of Vertebrates and Their Kin: The Macmillan Company.

Olson KR. 2002. Vascular anatomy of the fish gill. J Exp Zool 293:214-231.

Parichy DJ, Elizondo MR, Mills MG, Gordon TN, and Engeszer RE. 2009. Normal table of postembryonic zebrafish development: staging by externally visible anatomy of the living fish. Dev Dyn 238:2975-3015.

Passamaneck YJ, and Di Gregorio A. 2005. Ciona intestinalis: chordate development made simple. Dev Dyn 233:1-19. 10.1002/dvdy.20300

Ponec RJ. 1982. Natural heartbeat patterns of six ascidians and environmental effects on cardiac function in Clavelina huntsmani. Comp Biochem Physiol 72A:455-462.

Reiber CL, and McGaw IJ. 2009. A Review of the "Open" and "Closed" Circulatory Systems: New Terminology for Complex Invertebrate Circulatory Systems in Light of Current Findings. Inter J Zool 2009:. doi:10.1155/2009/301284.

Richardson PDI, and Granger DN. 1984. Microcirculation of the liver and spleen. In: Mortallaro NA, ed. The Physiology and Pharmacology of the Microcirculation. Orlando, FL USA: Academic Press, 95-131.

Ruppert EE. 1990. Structure, ultrastructure and function of the neural gland comple of Ascidia interrupta (Chordata, Ascidiacea): clsrification of hypothesis regarding the eveoution of the vertebrate anterior pituitary. Acta Zoologica (Stockholm) 71:135-149.

Ruppert EE, Fox RS, and Barns RD. 2004. Invertebrate zoology, a functional evolutionary approach. Belmont, CA USA: Thomson Brooks Cole.

Santhanakrishnan A, and Miller LA. 2011. Fluid dynamics of heart development. Cell Biochem Biophys 61:1-22. doi:10.1007/s12013-011-9158-8

Satoh N. 1994. Developmental Biology of Ascidians: Cambridge University Press.

Skramlik Ev. 1929. Uber den Kreislauf bei den Manteltieren. Z vergl Physiol 9:553-563.

Stach T, and Turbeville J, M. 2002. Phylogeny of Tunicata inferred from molecular and morphological characters. Molecular Phylogenetics and Evolution 2:408-428.

Stolfi A, Gainous TB, Young JJ, Mori A, Levine M, and Christiaen L. 2010. Early chordate origins of the vertebrate second heart field. Science 329:565-568.

Watkins SC, Maniar S, Mosher M, Roman BL, Tsang M, and St Croix CM. 2012. High Resolution Imaging of Vascular Function in Zebrafish. PLOS One 7:e44018. 


\section{Figure 1}

Figure 1. Tunicate stained with neutral red.

(A) Right side of animal; ventral at right, dorsal left, anterior top, posterior bottom. Scale bar equals $10 \mathrm{~mm}$. (B) Left side; animal rotated 180 degrees about vertical axis. Abbreviations: atrial - atrial siphon; oral - oral siphon; dbv - dorsal branchial vessel; vbv - ventral branchial vessel; orange strip - endostyle; arrows indicate blood flow during the branchial phase. 


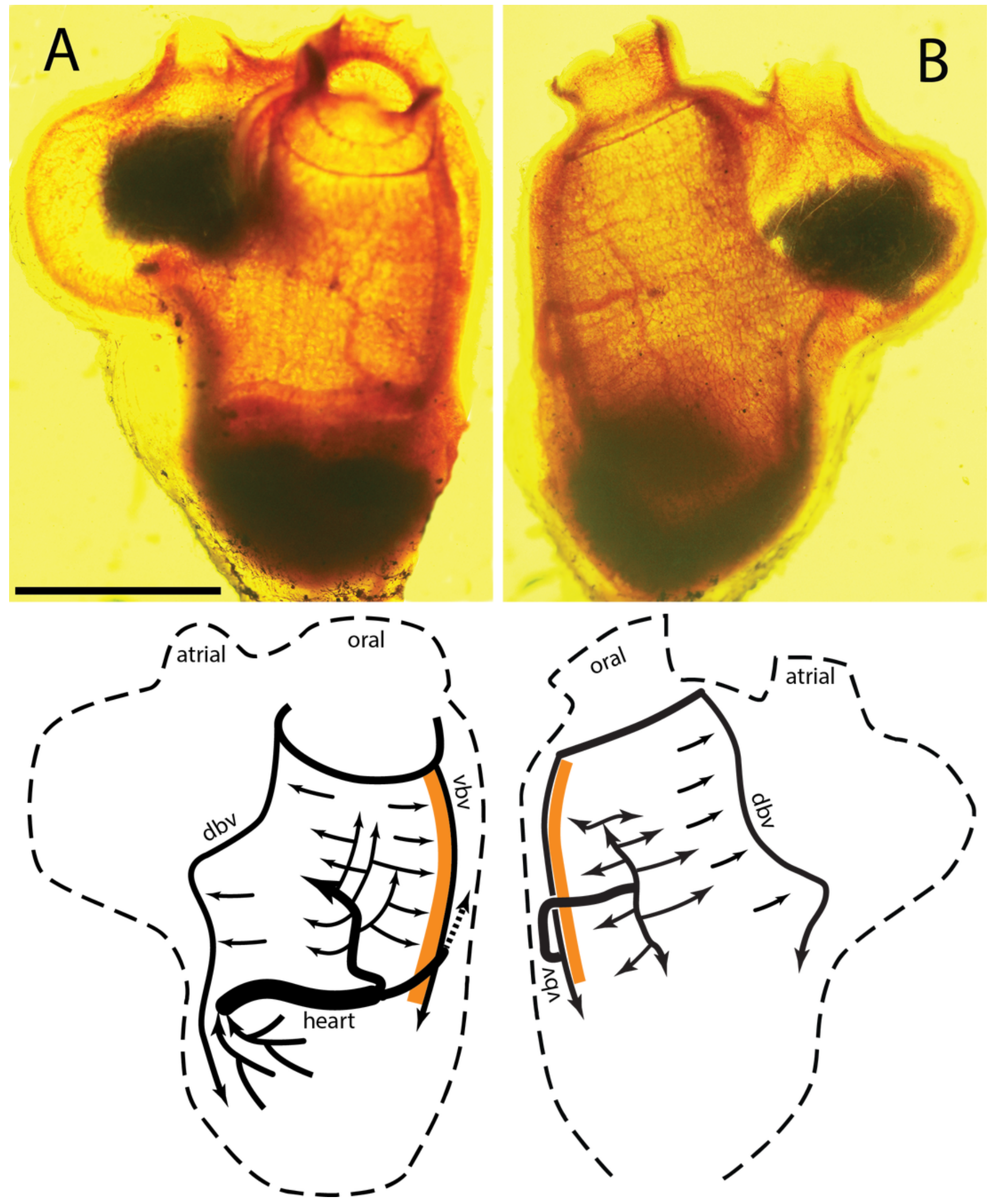




\section{Figure 2}

Figure 2. Flow of stained blood cells in the right side of the tunicate.

The ventral side of the tunicate is down and the anterior end is to the right. Blood leaves the heart at its ventral end and immediately splits into two vessels. Only the one supplying the right side of the branchial basket is shown here. After flowing through the branchial basket blood is collected by ventral and dorsal vessels and flows in the posterior direction into the viscera (green area marked by " $\mathrm{V}$ "). Blood is collected from the viscera in a network of small vessels that progressively merge to form two large vessels that enter the heart at its dorsal end. When the heart pumps in the posterior direction all flows are reversed. In the simplified diagram only the outer heart tube is drawn, and thus there is no constriction. Scale bar at lower left is $10 \mathrm{~mm}$. 


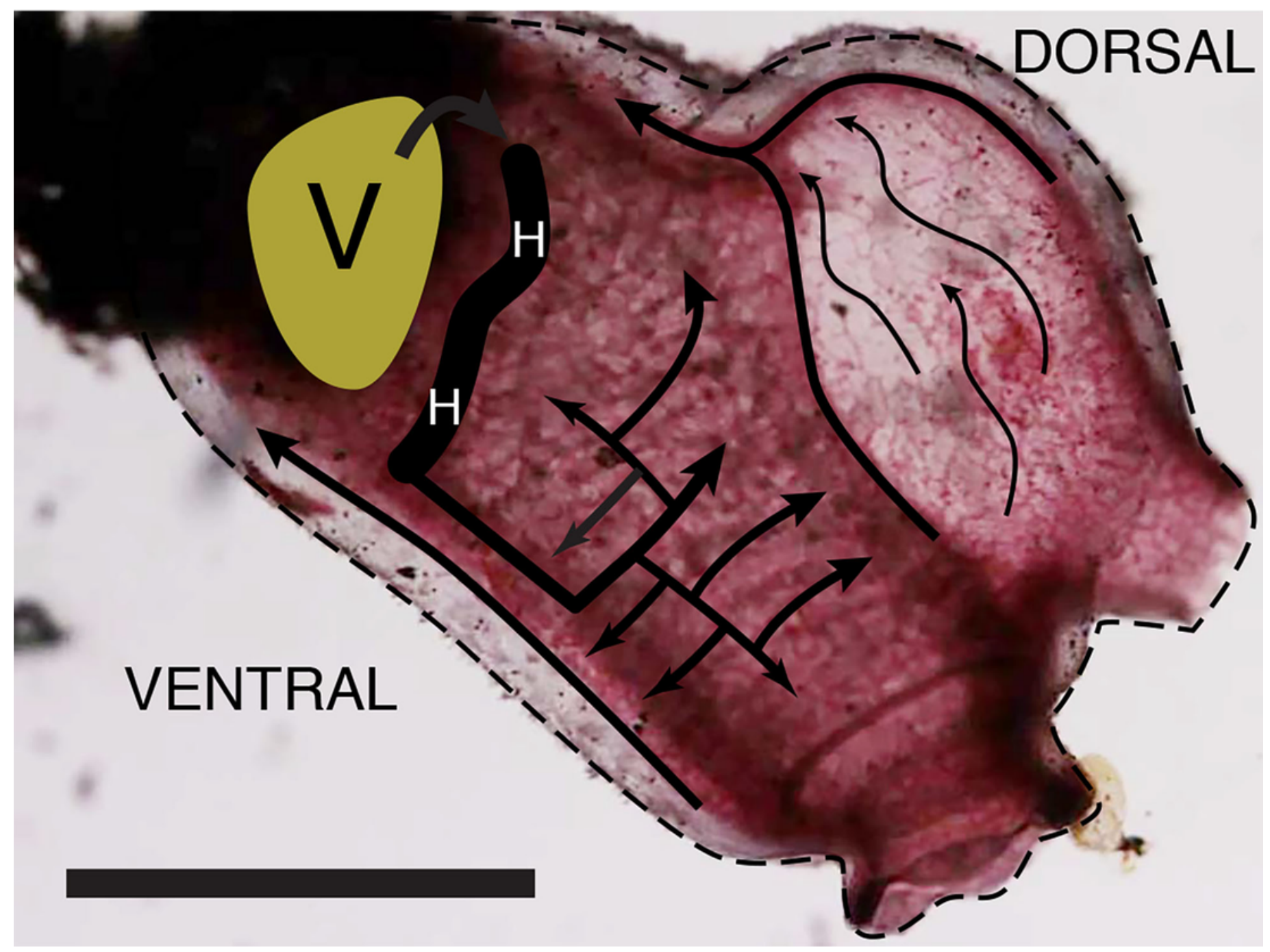




\section{Figure 3}

Figure 3. Florescent dextran injected into the circulatory system.

(A) Dextran injected (25 uL over $1.9 \mathrm{~min}$ ) at the posterior end of heart while the heart pumped in the branchial direction. This image was obtained $3.5 \mathrm{~min}$ after end of injection. Dextran enters the branchial basket through a branching tree of vessels in the side of the basket. (B) Dextran was injected (15 uL over $28 \mathrm{sec}$.) at the anterior end of heart while heart pumped in the visceral direction. The image was obtained $6.5 \mathrm{~min}$ after end of injection. Dextran leaves the heart in two large vessels that then branch repeatedly to supply the visceral region, e.g. stomach and gonads. Some blood flows into the dorsal vessel and then moves toward the anterior. Scale bar at bottom left represents $10 \mathrm{~mm}$.

*Note: Auto Gamma Correction was used for the image. This only affects the reviewing manuscript. See original source image if needed for review. 
A

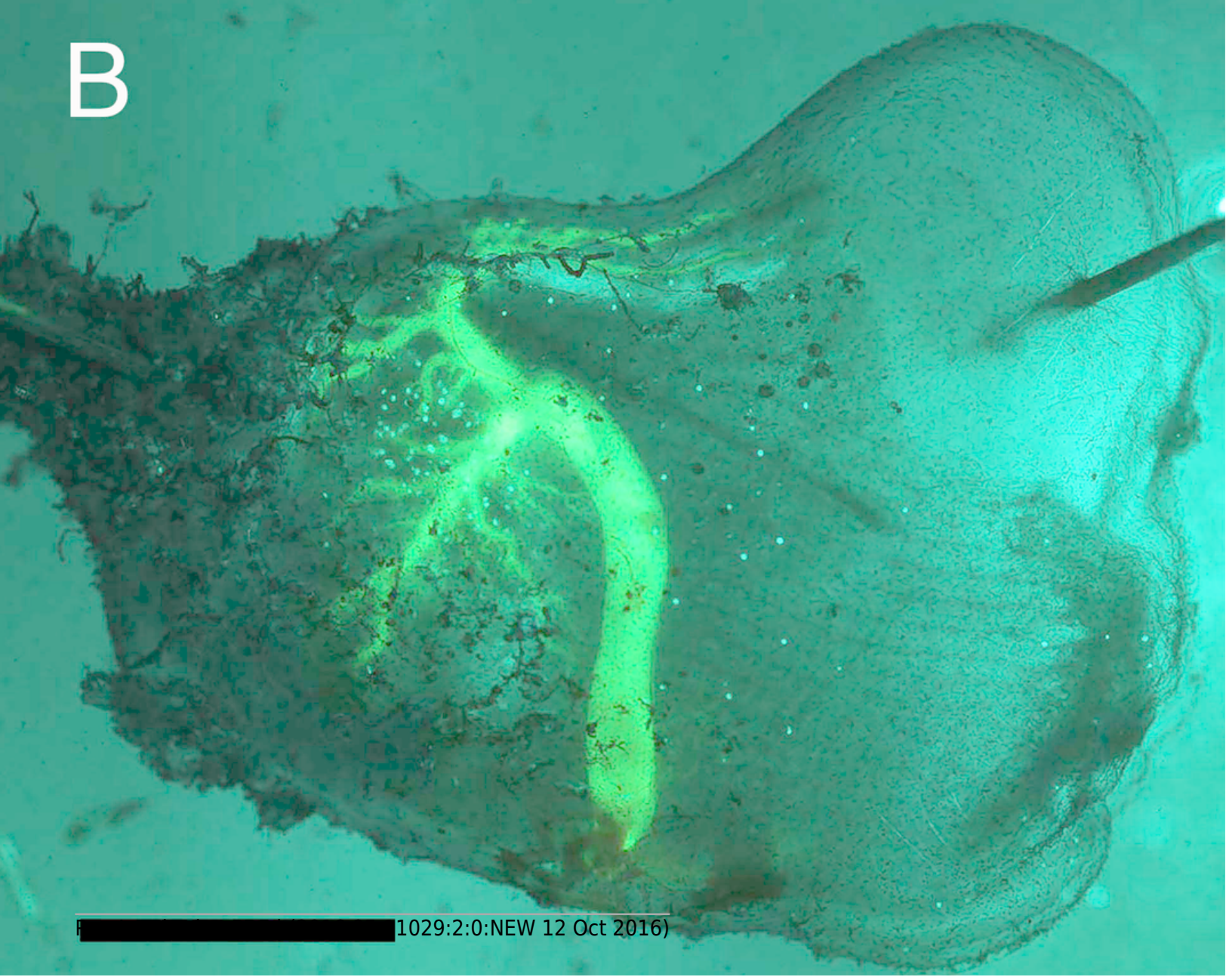




\section{Figure 4}

Figure 4. Fluorescent dextran injected into the pericardial space.

Image obtained more than 24 hours after the injection and the heartbeat was normal throughout this period. Ventral is at bottom and anterior is to the right; the scale bar at bottom represents $10 \mathrm{~mm}$. 


\section{Figure 5}

Figure 5. Folds in the internal heart tube at the central constriction.

At the top is a diagram of the ventral end of the heart and the vessel that connects to the right side of the branchial basket. The tunicate is rotated relative to previous figures, and the ventral edge is upward, anterior to the left. The " $\mathrm{V}$ " at upper right indicates the viscera. Segments of the heart tube containing blood on each side of the twist are labeled " $\mathrm{H}$ ", and the scale bar represents $2 \mathrm{~mm}$. The bottom panel is a photo of this region in a tunicate stained with neutral red. 

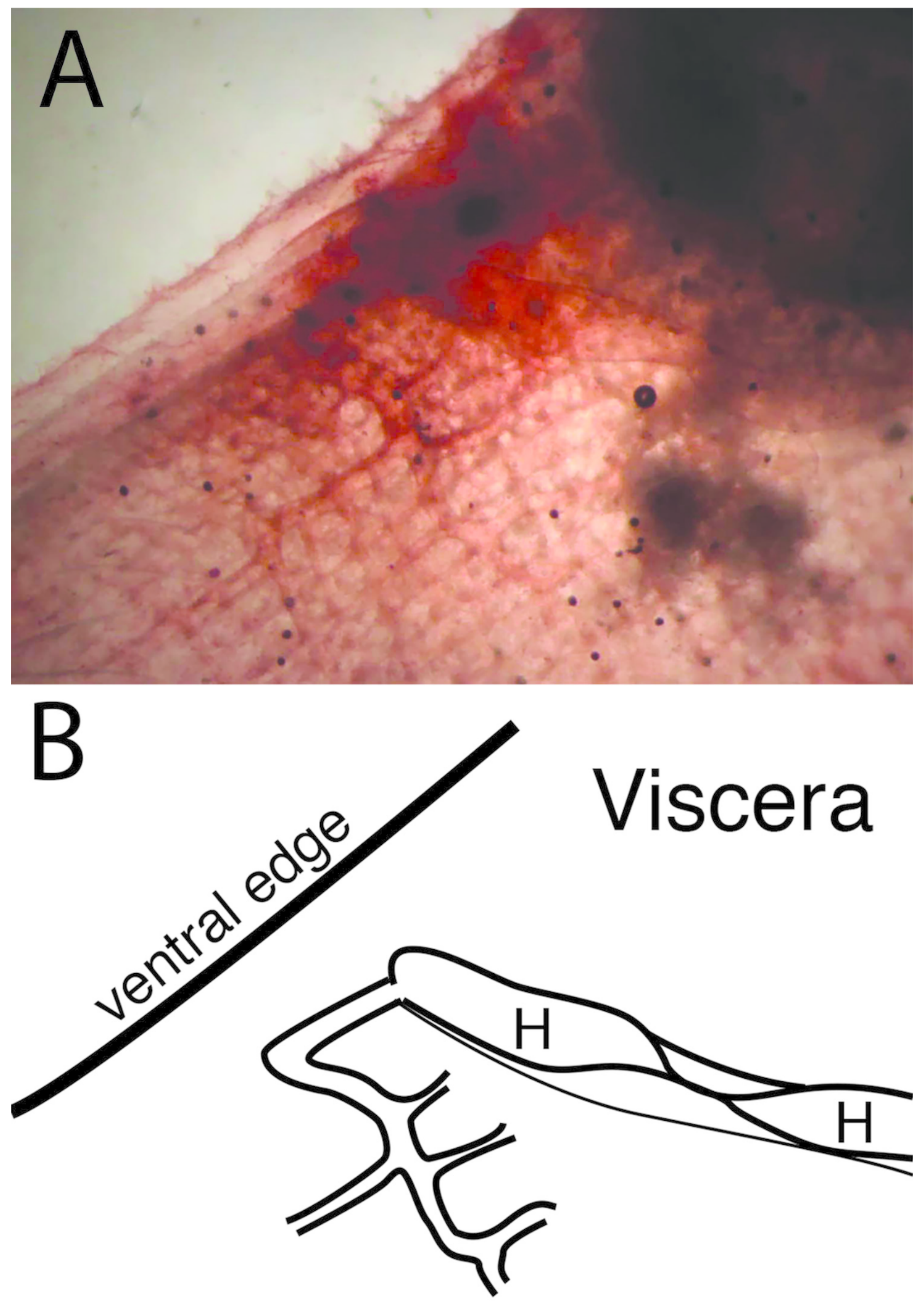


\section{Figure 6 (on next page)}

Figure 6. Velocities of blood cells in a large vessel.

A video with approximately the same field of view as Fig. 5 was disassembled into individual frames (30 per second), and the position of two densely stained cells was followed through sequential frames. Values in the graph are moving averages using Gaussian weighed values from five consecutive frames. The average blood cell velocities over the two complete heartbeats was approximately $0.9 \mathrm{~mm} / \mathrm{sec}$. 


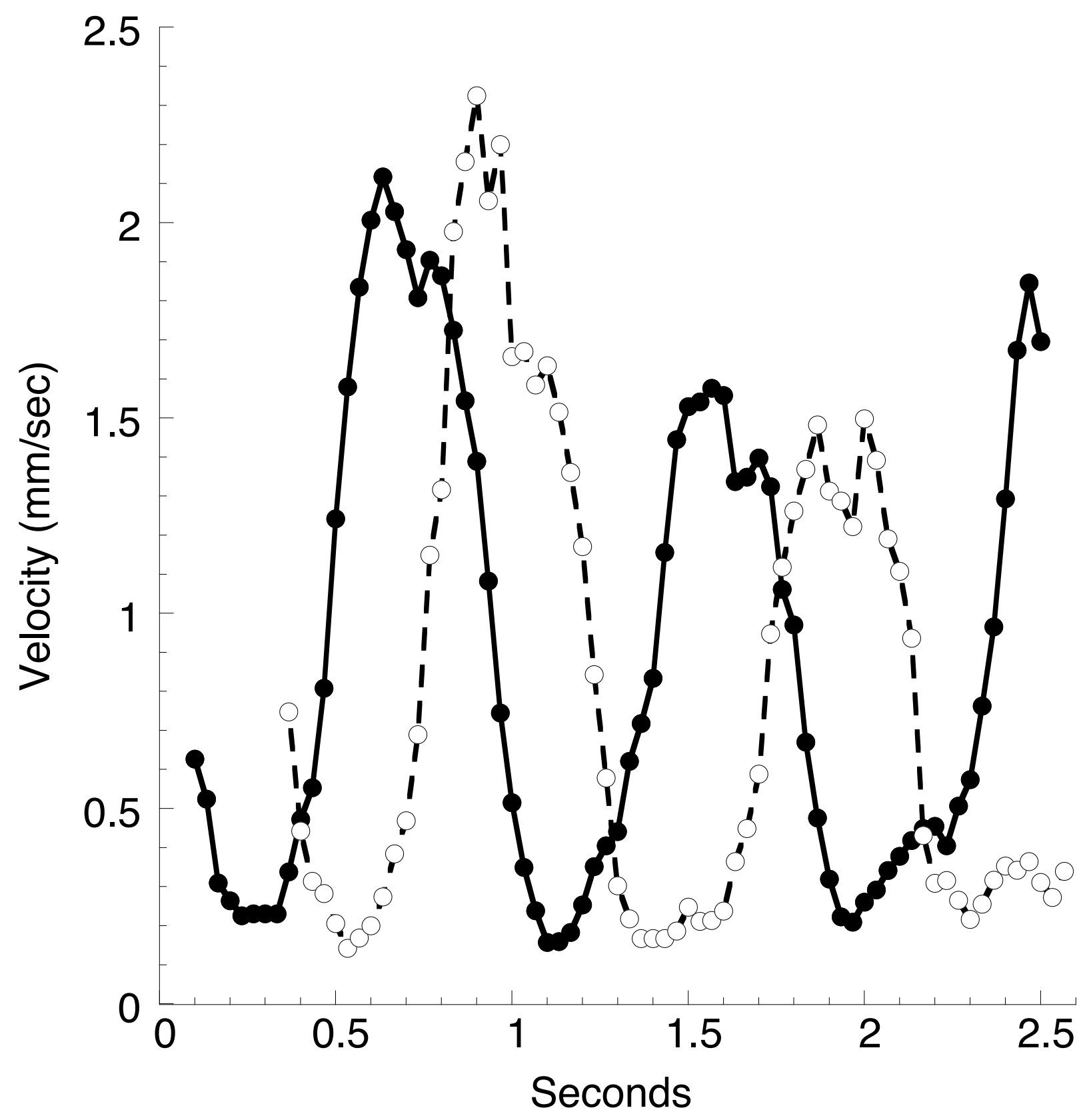


Figure 7 (on next page)

Velocities of blood cells in three small vessels.

A video of stained blood cells in the mantle surrounding the exit siphon was analyzed in the same manner as described in Fig. 6. 


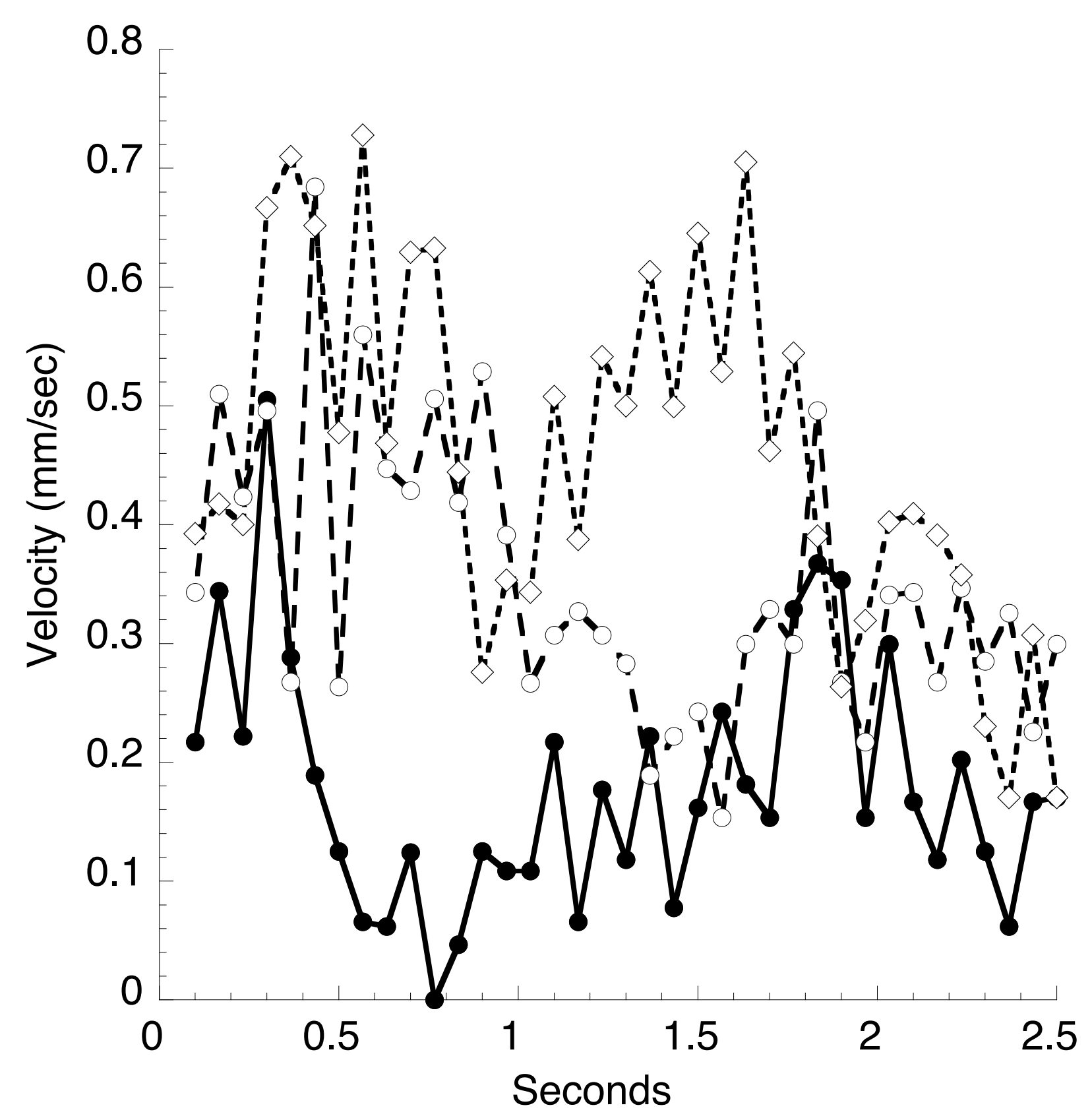




\section{Figure 8}

Layers of the branchial basket.

The axis of the tunicate is horizontal with anterior to the right. Height of view in each panel is $1 \mathrm{~mm}$. Each panel is from a different animal, but with corresponding positions and orientations. A: Interior surface (stained with neutral red). The two prominent horizontal bands in the Figure are the longitudinal bars of the basket. There are five vertical vessels perpendicular to each transverse bar that are under the longitudinal bars (only the two on the right are clearly defined in this image). B: The exterior surface of the basket (stained with neutral red). The spirals that resemble lonic volutes are the openings (stigmata) through which water is pumped. Higher magnification (not shown here) reveals the motion of beating cilia on the edge of the stigmata. C. Exterior surface after injection of fluorescent dextran posterior to heart. Blood flows through a complex network of ducts linked together with net circulation perpendicular to the axis of the tunicate. Scale bar at bottom left represents 1 $\mathrm{mm}$. 


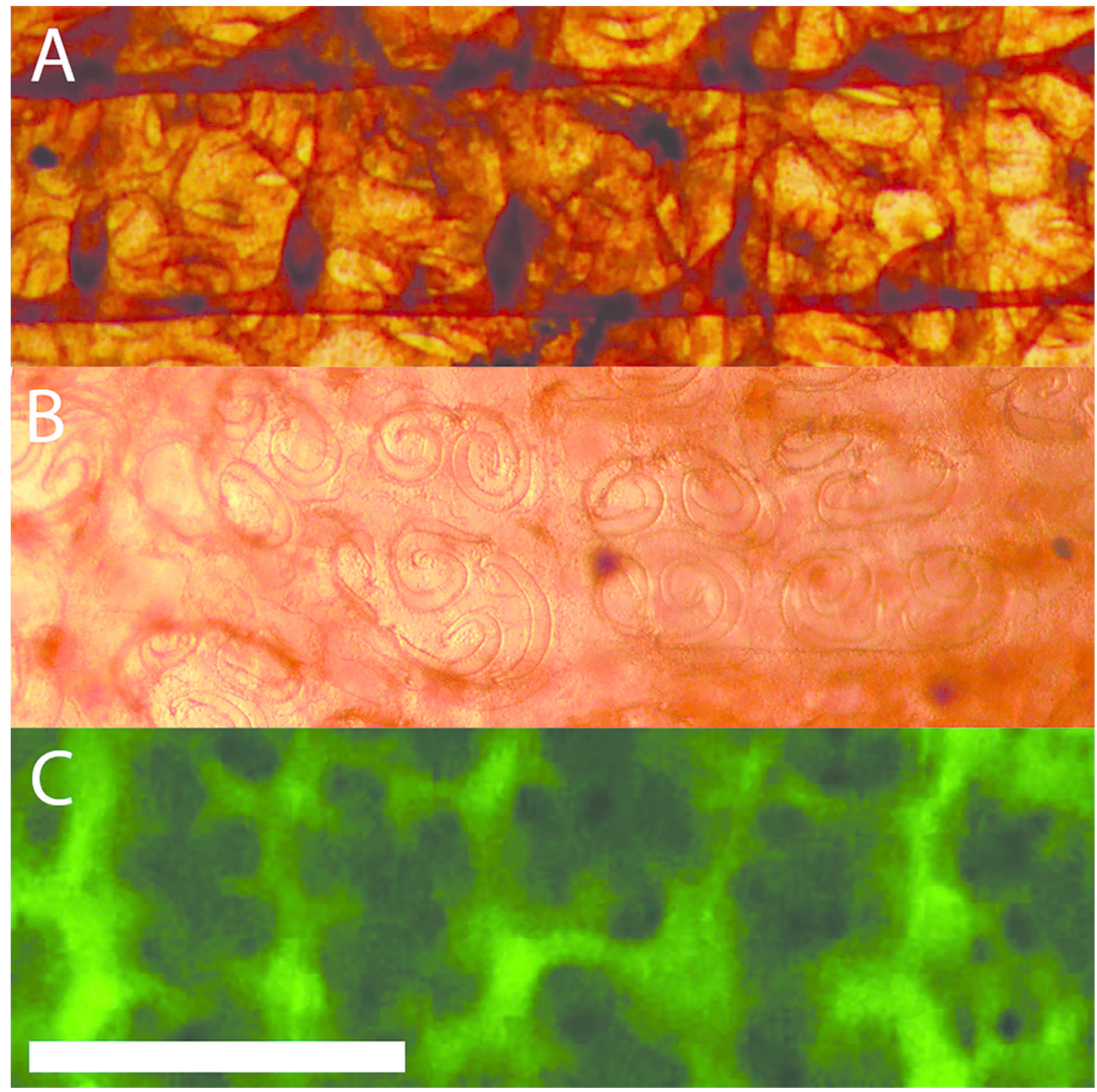




\section{Table $\mathbf{1}$ (on next page)}

Six consecutive heart beat phases of the peristaltic heart.

Visceral flow is from heart to viscera, branchial flow is from heart to the branchial basket. 
1

\begin{tabular}{|l|l|l|l|}
\hline Direction & Time (sec) & Heart beats & Beats/sec (Hz) \\
\hline visceral & 236 & 176 & 0.75 \\
\hline branchial & 318 & 220 & 0.69 \\
\hline visceral & 243 & 160 & 0.66 \\
\hline branchial & 293 & 197 & 0.67 \\
\hline visceral & 261 & 183 & 0.70 \\
\hline branchial & 252 & 170 & 0.67 \\
\hline visceral: avg (std dev) & $\mathbf{2 4 7 ( 1 3 )}$ & $\mathbf{1 7 3}(\mathbf{1 2})$ & $\mathbf{0 . 7 0}(\mathbf{0 . 0 5})$ \\
\hline branchial: avg (std dev) & $\mathbf{2 8 8 ( 3 3 )}$ & $\mathbf{1 9 6}(\mathbf{2 5})$ & $\mathbf{0 . 6 8}(\mathbf{0 . 0 1 )}$ \\
\hline
\end{tabular}

2 